\title{
Intervenir sur les TMS
}

Un modèle des Troubles Musculo-squelettiques comme objet intermédiaire entre ergonomes et acteurs de l'entreprise?

Preventing musculoskeletal disorders. Can an MSD model be used as an intermediary object between ergonomists and company actors?

Willy Buchmann et Aurélie Landry

\section{(2) OpenEdition}

Journals

Édition électronique

URL : http://journals.openedition.org/activites/2418

DOI : 10.4000/activites. 2418

ISSN : $1765-2723$

Éditeur

ARPACT - Association Recherches et Pratiques sur les ACTivités

Référence électronique

Willy Buchmann et Aurélie Landry, «Intervenir sur les TMS », Activités [En ligne], 7-2 I octobre 2010, mis en ligne le 15 octobre 2010, consulté le 19 avril 2019. URL : http://journals.openedition.org/ activites/2418 ; DOI : 10.4000/activites. 2418

\section{(c) (i) (9)}

Activités est mis à disposition selon les termes de la licence Creative Commons Attribution - Pas d'Utilisation Commerciale - Pas de Modification 4.0 International. 


\title{
Intervenir sur les TMS. \\ Un modèle des Troubles Musculo-squelettiques comme objet intermédiaire entre ergonomes et acteurs de l'entreprise ?
}

\author{
Willy Buchmann \\ CREAPT \\ Immeuble Le Descartes I - 29 promenade Michel-Simon - 93166 Noisy-le-Grand Cedex, France \\ willybuchmann@aol.com
}

\author{
Aurélie Landry \\ PACTE, Université de Grenoble \\ Le Patio - 1041 rue des Universités, BP 47 - 38040 Grenoble Cedex 9, France \\ Aurelie.landry@laposte.net
}

\begin{abstract}
Preventing musculoskeletal disorders. Can an MSD model be used as an intermediary object between ergonomists and company actors? A multiple-case study of ergonomics projects aimed at preventing musculoskeletal disorders (MSDs) led us to test whether the use of a model was desirable to ensure sustainable MSD prevention in companies. Once an MSD model incorporating both MSD etiology and the prevention project has been selected, according to the theory of "intermediate object", the use of an MSD model would seem to facilitate discussions among company actors concerned with MSDs. Moreover, the model seems to give them a better understanding of MSDs, which in turn encourages them to make suggestions for adjusting the model to suit their company. After using an MSD model, the authors propose further developments by incorporating both new information about MSD etiology and the adjustments put forward by the company actors.
\end{abstract}

\section{KEYWORDS}

operating model, intermediate object, musculoskeletal disorders, sustainable prevention.

L'objet de cet article est de préciser, à l'aide d'une étude qualitative de cas multiples, si un modèle des troubles musculo-squelettiques (TMS) peut servir de support entre les intervenants et les acteurs d'une entreprise pour organiser les actions de prévention et réduire l'exposition aux facteurs de risques de TMS. Pour cela nous avons utilisé la méthode proposée par Yin (1994) et Baril-Gingras, Bellemare et Brun (2006) consistant à analyser des données recueillies par observations et entretiens, ainsi que le contenu de documents de manière à identifier les effets de l'usage d'une représentation d'un problème (ici les TMS) sur les acteurs participants à une intervention visant à leur prévention. En effet Aptel et Vézina (2008) rappellent qu'un modèle d'intervention ergonomique et de la problématique TMS est un moyen d'action opérationnelle pour l'ergonome afin de convaincre les acteurs sociaux et les responsables d'entreprise « du bien fondé d'entreprendre des actions de prévention des TMS ». L'utilisation d'un modèle et l'importance de son partage avec les acteurs de l'entreprise rejoint les constats de Bellemare, Marier, Montreuil, Allard et Prévost (2002), de Denis, St Vincent, Jetté, Nastasia et Imbeau (2005) et de Caroly, Coutarel, Escriva, Roquelaure et Schweitzer (2007) en ce qui concerne les interventions en ergonomie visant la prévention des TMS. D'après les auteurs (ibid.), l'intervenant doit dans la mesure du possible, se doter d'outils permettant de :

- engager et construire socialement la prévention,

- constituer un collectif dans les équipes de prévention (ergonome, médecin du travail, infirmière, 
responsable sécurité...),

- dialoguer avec les décideurs, les responsables syndicaux,

- créer une dynamique autour des pôles « pouvoir penser », « pouvoir débattre » et « pouvoir agir » (Daniellou, 1998).

Pour atteindre ces objectifs, l'intervenant peut donc avoir recours à différents instruments, vus ici comme des objets intermédiaires à partager (Jeantet, 1998). Une représentation de l'étiologie des TMS et des étapes de l'intervention que nous appellerons par la suite «modèle des TMS » pourrait être un support à la construction sociale de l'intervention et à son déroulement tout en respectant les qualités scientifiques du problème posé (Aptel \& Vézina, 2008, op. cit.). C'est ce que nous souhaitons étudier à travers l'étude de cas multiples relatée dans cet article.

\section{1.- Démarche mise en place favorisant la mise en débat de la santé au travail}

\section{1.- Question de recherche et démarche utilisée}

Notre démarche cherche à répondre à la question suivante : est-ce qu'un modèle des troubles musculo-squelettiques (TMS) peut servir de référentiel commun entre les intervenants et les acteurs d'une entreprise ? C'est-à-dire est-ce qu'un modèle des TMS tel que nous en trouvons dans la littérature peut être facilement appropriable par les acteurs des entreprises pour mettre en débat la santé au travail et engager des démarches de prévention?

Ces questions placent notre recherche dans la compréhension des effets produits par des modalités d'intervention (Landry, 2008). La figure 1 issue d'une proposition de St Vincent, Gonella, Beauvais, Vézina, Laberge, Lévesque et al. (2008) précise que nous nous intéresserons aux méthodes et outils qu'un intervenant utilise lors de son intervention à l'occasion de groupes projet, groupes de travail... et que nous cherchons à mettre en lien ces choix méthodologiques avec les effets produits sur les représentations des acteurs de l'entreprise.

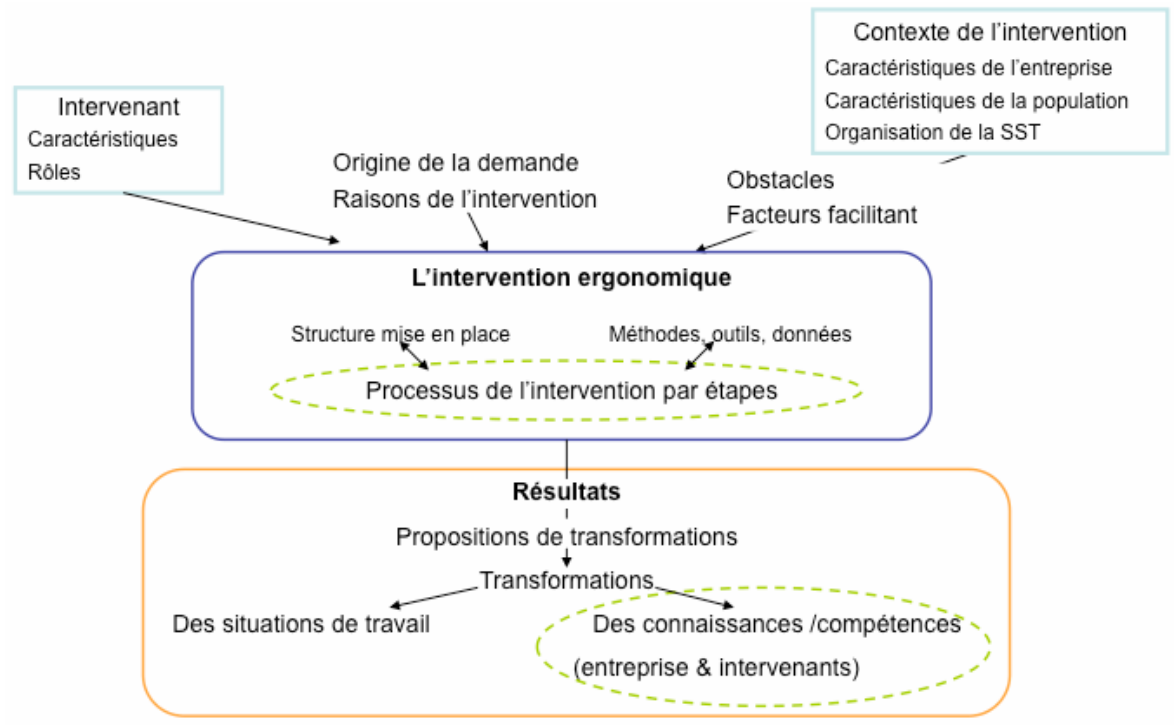

Figure 1 : Modèle de l'intervention, des facteurs qui l'influencent et des résultats produits (tiré de St Vincent, Gonella, Beauvais, Vézina, Laberge, Lévesque et al., 2008).

Figure 1: Model of factors influencing the course of projects and the results (from St Vincent, Gonella, Beauvais, Vézina, Laberge, Lévesque et al., 2008). 
Cette modélisation de l'intervention et des facteurs qui l'influencent montrent également qu'il est important de tenir compte du contexte de l'intervention (caractéristiques de l'entreprise, de la population, organisation de la SST) et des caractéristiques de l'intervenant pour comprendre les effets produits.

Ainsi pour répondre à notre question de recherche, nous avons choisi de comparer 3 interventions (Baril-Gingras, Bellemare, \& Brun 2006, op. cit.; Leplat, 2002) qui se sont déroulées dans 3 contextes différents mais où les intervenants ont adopté la même stratégie d'action : partager avec les acteurs de l'entreprise une modélisation des TMS. Ces interventions sont réalisées par des ergonomes en thèse, ils ont pour mission de réaliser des actions en entreprises, tout en adoptant une posture de recherche afin d'utiliser les interventions réalisées comme données de recherche.

\section{2.- Présentation des caractéristiques des entreprises}

Avant de présenter le cadre théorique sur lequel nous nous appuierons pour analyser les effets produits par les choix de méthodologies d'intervention, nous allons détailler le contexte dans lequel les interventions se sont déroulées. Nous nous appuyons sur le recensement par St Vincent et al. (2008, $o p$. cit.) des facteurs qui peuvent influencer l'intervention dans le choix des méthodes et ainsi influencer les résultats produits. Le tableau 1 reprend de manière synthétique les caractéristiques des interventions étudiées.

\begin{tabular}{|c|c|c|c|c|c|c|c|c|}
\hline 象 & 竝 & 节 & 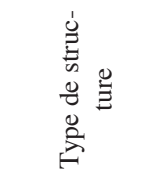 & 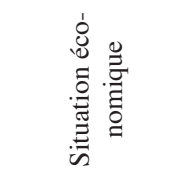 & 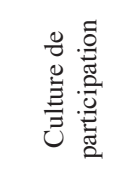 & 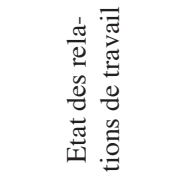 & 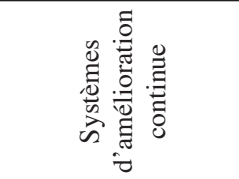 & 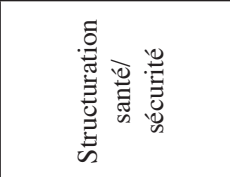 \\
\hline $\mathbf{A}$ & $\begin{array}{l}\text { Grande d } \\
\text { istribution }\end{array}$ & $\begin{array}{c}50 \\
\text { salariés }\end{array}$ & $\begin{array}{c}\text { Site appar- } \\
\text { tenant à un } \\
\text { groupe national }\end{array}$ & $\begin{array}{c}\text { Très concur- } \\
\text { rentielle bonne } \\
\text { situation écono- } \\
\text { mique }\end{array}$ & $\begin{array}{l}\text { Peu partici- } \\
\text { pative }\end{array}$ & $\begin{array}{c}\text { Relations } \\
\text { tendues entre } \\
\text { les différents } \\
\text { niveaux hiérar- } \\
\text { chiques }\end{array}$ & $\begin{array}{l}\text { Amélioration continue } \\
\text { du chiffre de vente }\end{array}$ & $\begin{array}{c}\text { Aucunes ressources } \\
\text { en interne }\end{array}$ \\
\hline B & $\begin{array}{l}\text { Montage } \\
\text { électrique }\end{array}$ & $\begin{array}{l}1500 \\
\text { salariés }\end{array}$ & $\begin{array}{l}\text { Site appar- } \\
\text { tenant à un } \\
\text { groupe interna- } \\
\text { tional }\end{array}$ & Concurrentielle & Participative & $\begin{array}{l}\text { Prise en compte } \\
\text { des aspects } \\
\text { sécurité et santé } \\
\text { au travail }\end{array}$ & $\begin{array}{l}\text { Certification qualité et } \\
\text { environnement. } \\
\text { Système de manage- } \\
\text { ment de la sécurité }\end{array}$ & $\begin{array}{l}\text { Service prévention : } \\
\text { Infirmières } \\
\text { Médecins du travail }\end{array}$ \\
\hline
\end{tabular}

Tableau 1 : Présentation des principales caractéristiques des entreprises dans lesquelles se sont déroulées les interventions.

Table 1: Main characteristics of the companies involved in the three ergonomic projects.

L'entreprise A est un établissement de type supermarché. Il appartient à un groupe national qui transfère les modes d'organisation de la vente (surface de vente, surface de stock, modes de travail) à tous les magasins de France. La grande distribution est un secteur très concurrentiel notamment par rapport à l'implantation géographique des magasins dans les villes. Dans l'entreprise A, le mode de décision n'est pas participatif. Le directeur de magasin reçoit des consignes de la part d'un directeur régional qui a lui-même reçu des directives de la part d'une cellule de travail nationale. Le directeur de magasin applique les consignes tout en recherchant des marges de manœuvre pour s'adapter aux 
particularités des clients et des produits vendus. Pour cela il met à contribution les responsables de secteurs en discutant certains modes de travail. Cependant, les responsables de secteurs ne sont pas habitués à être consultés et la décision finale revient au directeur de magasin. Dans ce contexte, les salariés ne sont jamais associés aux changements. Cet arbitrage nécessaire pour rentrer dans les critères d'évaluations de la cellule nationale (des contrôles surprises ont lieu avec « des clients mystères ») conduisent à un climat tendu entre chaque niveau hiérarchique. La performance est calculée sur le chiffre de vente du magasin d'un mois sur l'autre et les consignes poussent à l'augmentation de ce chiffre de vente, sans tenir compte des variations saisonnières (vacances) qui peuvent faire diminuer le chiffre. En matière de sécurité et santé au travail, c'est le directeur de magasin qui doit effectuer les investissements ou les réparations en prenant sur son chiffre d'affaire. Les salariés du magasin sont suivis par un médecin du travail en service interentreprises.

L'entreprise $B$ est une entreprise française à renommée internationale spécialisée dans l'approvisionnement en électricité. L'intervention s'est déroulée dans plusieurs établissements d'une même ville où l'entreprise est implantée. L'activité est concurrentielle, l'entreprise doit proposer à ses clients des nouveaux produits pour maintenir sa place de leader sur le marché. Pour ce qui concerne la fabrication, la participation des opérateurs et des différents niveaux hiérarchiques est organisée dans la conduite de projet. Les effets des modes de fabrications sur la santé et la sécurité sont intégrés à la réflexion. Cependant les acteurs de l'entreprise souhaitent prioriser les actions de prévention et si possible intégrer la réflexion sur les conditions de réalisation du travail le plus en amont possible. Les sites de fabrications et d'innovations sont engagés dans des démarches d'amélioration continue de la qualité, de l'environnement et de la sécurité. Pour animer les démarches sécurité, l'entreprise est dotée d'un service prévention composé principalement d'infirmières du travail et de médecins du travail. Tous les sites ne disposent pas de médecins du travail qui coordonnent leurs actions avec les infirmières.

L'établissement $C$ est le plus petit de deux établissements français d'une entreprise spécialisée dans l'aéronautique, et filiale d'un grand groupe aéronautique européen. L'établissement C est situé en Île de France. Il est spécialisé dans la conception, la production et la réparation de pièces pour hélicoptères.

La production du site est assurée par 650 salariés dont 450 techniciens et ouvriers. L'effectif global est stable depuis 3 ans.

Les carnets de commandes de pièces sont remplis pour les 2 années à venir, et la quantité de pièces livrées augmente régulièrement depuis 2004 (+30 \% entre 2004 et 2008). Les orientations prises par la direction de production sont centrées sur la productivité et sur la tenue de tableaux de suivi de production. Dans ce contexte où les évolutions (processus de fabrication, moyens de travail) pour faire face aux augmentations de charge sont fréquentes et rapides, la santé / sécurité n'est prise en compte que par le respect des normes. Les Instances Représentatives du Personnel et les opérateurs ne sont souvent qu'informés.

Le système de santé-sécurité du site est organisé d'une part autour du service de santé au travail, interne à l'établissement, composé d'un médecin du travail (à temps complet), d'un infirmier (à temps complet), et d'autre part d'un service prévention des risques composé d'un responsable prévention, d'un animateur sécurité et de deux ingénieurs environnement.

L'établissement dispose d'un service dédié à l'amélioration de la compétitivité et d'un service d'amélioration qualité produit. Les ateliers de production font l'objet d'une démarche d'amélioration de la compétitivité de type Lean Manufacturing, et un cabinet de consultants en organisation anime la mise en place et le suivi de chantiers type Kaizen.

Ainsi, les caractéristiques des entreprises où les interventions ont été mises en œuvre différent sur la taille, le secteur d'activité, la dépendance vis-à-vis d'un groupe... Mais aussi sur un ensemble de facteurs identifiés comme favorables ou défavorables pour le déroulement des interventions en ergo- 
nomie (St Vincent et al., 2008, op. cit.) :

- la concurrence du marché,

- la culture de la participation,

- le climat des relations entre les différents niveaux hiérarchiques,

- l'existence de démarches d'améliorations continues,

- la présence de ressources internes en santé et sécurité au travail.

Contrairement à l'entreprise C, les entreprises A et B doivent innover pour maintenir leur place sur le marché, elles font de gros efforts dans ce sens, ce qui conduit à des changements fréquents dans le travail des salariés. Ce facteur a été identifié par St Vincent et al., 2008, op . cit., comme étant fréquemment un obstacle à la réalisation d'actions de prévention.

Contrairement à l'entreprise B, les entreprises $\mathrm{A}$ et $\mathrm{C}$ n'ont pas une culture de participation favorable à l'implication des salariés dans le changement, de même dans ces entreprises le climat des relations entre les différents niveaux hiérarchiques est plutôt tendu. Ces caractéristiques semblent avoir une influence négative sur les actions en ergonomie.

Les 3 entreprises sont impliquées dans des démarches d'amélioration continue, cependant seule l'entreprise $\mathrm{B}$ intègre les questions de sécurité et de prévention à ces systèmes. Ceci pouvant avoir une influence positive sur la mise en œuvre de l'intervention en ergonomie. Enfin, contrairement à l'entreprise A, les entreprises B et C peuvent appuyer leur démarche sur des ressources internes en santé et sécurité ce qui est plutôt favorable aux actions en ergonomies (Baril-Gingras et al., 2004, op. cit.).

Ces caractéristiques et leurs influences sont donc réparties parmi les cas (Tableau 2). Ils pourront jouer un rôle sur les effets produits par l'intervention.

\begin{tabular}{|c|c|c|c|c|c|}
\hline & $\begin{array}{l}\text { Concurrence du } \\
\text { marché faible }\end{array}$ & $\begin{array}{l}\text { Culture de la } \\
\text { participation } \\
\text { existante }\end{array}$ & $\begin{array}{l}\text { Climat de } \\
\text { relations } \\
\text { favorables }\end{array}$ & $\begin{array}{l}\text { Démarches } \\
\text { d'améliorations } \\
\text { continues santé }\end{array}$ & $\begin{array}{c}\text { Existences de } \\
\text { Ressources internes en } \\
\text { santé/Sécurité }\end{array}$ \\
\hline \multicolumn{6}{|l|}{ A } \\
\hline B & & $\mathrm{x}$ & $\mathrm{x}$ & $\mathrm{x}$ & $\mathrm{x}$ \\
\hline C & $\mathrm{x}$ & & & & $\mathrm{X}$ \\
\hline
\end{tabular}

Tableau 2 : Récapitulatif des caractéristiques des entreprises favorables à l'action en santé.

Table 2: Summary of the characteristics of companies that support health projects.

Ainsi, la comparaison inter-cas (Leplat, 2002) cherchera à identifier les effets produits par l'intervention compte tenu des caractéristiques du contexte des entreprises. Les influences du contexte sur les effets observés pour un cas pourront être contrebalancées par les effets produits pour les deux autres cas. La répétition inter-cas des effets produits par l'intervention cherche à isoler les effets de ces variables (Yin, 1994, op. cit.) par répétition artificielle (Falzon, 1996).

\section{3.- Présentation des caractéristiques des interventions}

Comme nous l'avons indiqué nous nous intéressons aux effets produits par un choix d'intervention. Afin de préciser les caractéristiques de l'intervention, nous avons synthétisé pour chaque entreprise les caractéristiques des intervenants, la demande, les méthodes utilisées et les structures d'interventions mises en place (c'est-à-dire la façon dont la démarche est participative ou non) (Landry, 2008, op. cit.; St Vincent et al., 2008, op. cit.). Nous précisons également pour chaque cas, quels sont les leviers et obstacles en plus des éléments structurels détaillés dans le tableau 1 qui interfèrent sur le déroulement de l'intervention d'après les ergonomes (Landry \& Tran Van, 2010) et qui ont pu 
influencer les choix méthodologiques d'intervention ainsi que les résultats produits.

\begin{tabular}{|c|c|c|c|c|c|c|c|}
\hline שี & Demande & $\begin{array}{l}\text { Caractéristiques } \\
\text { intervenants }\end{array}$ & Méthodes & $\begin{array}{c}\text { Structures } \\
\text { d'interven- } \\
\text { tion }\end{array}$ & $\begin{array}{c}\text { Données re- } \\
\text { cueillies }\end{array}$ & Leviers & $\begin{array}{l}\text { Obstacles à l'inter- } \\
\text { vention }\end{array}$ \\
\hline A & $\begin{array}{c}\text { Accompagnement } \\
\text { d'un projet de } \\
\text { conception et } \\
\text { prise en compte } \\
\text { de la prévention } \\
\text { des TMS }\end{array}$ & $\begin{array}{l}\text { Ergonome } \\
\text { chercheur / re- } \\
\text { cherche-action }\end{array}$ & $\begin{array}{l}\text { Construction } \\
\text { d'un référentiel } \\
\text { commun en } \\
\text { utilisant une } \\
\text { modélisation } \\
\text { des TMS }\end{array}$ & $\begin{array}{l}\text { Groupe pro- } \\
\text { jet et comité } \\
\text { de suivi }\end{array}$ & $\begin{array}{c}\text { Analyses de } \\
\text { l'activité de } \\
\text { travail et entre- } \\
\text { tiens }\end{array}$ & $\begin{array}{c}\text { Disponibilité } \\
\text { du directeur de } \\
\text { magasin } \\
\text { Collaboration } \\
\text { des opérateurs et } \\
\text { managers }\end{array}$ & $\begin{array}{l}\text { Peu de marges de } \\
\text { manœuvre écono- } \\
\text { miques pour la trans- } \\
\text { formation } \\
\text { Décisions relevant } \\
\text { d'un siège social }\end{array}$ \\
\hline B & $\begin{array}{l}\text { Priorisation des } \\
\text { secteurs de pré- } \\
\text { vention par rap- } \\
\text { port aux risques } \\
\text { TMS }\end{array}$ & $\begin{array}{l}\text { Ergonome cher- } \\
\text { cheur et ergo- } \\
\text { nome consultant }\end{array}$ & $\begin{array}{l}\text { Construction } \\
\text { d'un référentiel } \\
\text { commun en } \\
\text { utilisant une } \\
\text { modélisation } \\
\text { des TMS }\end{array}$ & $\begin{array}{l}\text { Groupe pro- } \\
\text { jet (service } \\
\text { prévention) }\end{array}$ & $\begin{array}{c}\text { Analyses de } \\
\text { l'activité de } \\
\text { travail et entre- } \\
\text { tiens }\end{array}$ & $\begin{array}{c}\text { Présence d'un } \\
\text { service préven- } \\
\text { tion } \\
\text { Collaboration } \\
\text { des opérateurs } \\
\text { Support de la } \\
\text { direction }\end{array}$ & $\begin{array}{l}\text { Volumes de produc- } \\
\text { tion en diminution }\end{array}$ \\
\hline $\mathrm{C}$ & $\begin{array}{l}\text { Prévention des } \\
\text { TMS dans un } \\
\text { projet de concep- } \\
\text { tion de } 2 \text { postes } \\
\text { de travail }\end{array}$ & $\begin{array}{l}\text { Ergonome cher- } \\
\text { cheur interne } \\
\text { à l'entreprise } \\
\text { (CIFRE) }\end{array}$ & $\begin{array}{l}\text { Construction } \\
\text { d'un référentiel } \\
\text { commun en } \\
\text { utilisant une } \\
\text { modélisation } \\
\text { des TMS }\end{array}$ & $\begin{array}{l}\text { Groupe pro- } \\
\text { jet (Bureau } \\
\text { d'études) }\end{array}$ & $\begin{array}{l}\text { Analyses de } \\
\text { l'activité de } \\
\text { travail sur } \\
\text { postes simi- } \\
\text { laires dans } \\
\text { l'entreprise et } \\
\text { entretiens } \\
\text { Normes er- } \\
\text { gonomiques } \\
\text { européennes }\end{array}$ & $\begin{array}{l}\text { Demande d'as- } \\
\text { sistance formu- } \\
\text { lée tôt par le } \\
\text { chef de projet } \\
\text { Réunions régu- } \\
\text { lières avec le } \\
\text { chef de projet } \\
\text { Collaboration } \\
\text { des opérateurs et } \\
\text { managers }\end{array}$ & $\begin{array}{c}\text { Nécessité d'inno- } \\
\text { ver (anciens postes } \\
\text { >20ans : reproduc- } \\
\text { tibilité impensable, } \\
\text { et faible poids des } \\
\text { situations de réfé- } \\
\text { rence) } \\
\text { Marges de manœuvre } \\
\text { économiques faibles, } \\
\text { puis revues à la } \\
\text { baisse } \\
\text { Projet lancé puis } \\
\text { gelé à } 2 \text { reprises en } \\
2 \text { ans, changement } \\
\text { des acteurs au fil du } \\
\text { projet }\end{array}$ \\
\hline
\end{tabular}

Tableau 3 : Caractéristiques des interventions selon les 3 entreprises.

Table 3: Characteristics of the ergonomic projects in the three companies.

Dans l'entreprise A, l'intervention consiste en l'accompagnement d'un projet de conception (Landry $\&$ Feillou, 2008). Il est demandé aux ergonomes d'intégrer la prévention des TMS à l'occasion d'une réflexion sur le concept de vente qui se traduit en un réaménagement des locaux. L'intégration de la prévention des TMS a lieu à la fois en proposant des recommandations sur les situations de travail et sur le déroulement d'un projet de conception architecturale. Le projet est piloté par un groupe projet constitué d'un directeur de magasin et des responsables de secteur. Un comité de suivi est également mis en place pour suivre à une fréquence moindre le déroulement du projet, celui-ci est constitué du directeur de magasin, du médecin du travail et du responsable régional. Des groupes de travail sont également mis en place avec les opérateurs pour instruire les cahiers des charges et simulations mises en place lors de l'intervention. Avant le démarrage des analyses, les ergonomes choisissent de lancer le projet en construisant un référentiel commun sur la démarche en ergonomie et la problématique TMS. Pour cela, ils utilisent donc un modèle explicatif qu'ils commentent en groupe projet et en comité de suivi. Par la suite, les données recueillies (analyses de l'activité, entretiens) vont illustrer le modèle avec les particularités du travail effectué dans le magasin. Les autres modélisations concernent les circulations et les flux, ainsi que des outils de simulations de l'activité future.

Les leviers identifiés par les ergonomes dans cette intervention relèvent de l'implication et de la disponibilité des différents acteurs du magasin. Le directeur est impliqué et se rend disponible pour des 
échanges fréquents avec les ergonomes. Les responsables de secteurs et les opérateurs s'impliquent également dans la recherche de solution. L'intervention est également soutenue (Landry \& Tran Van, 2010, op . cit.) par la présence sur site d'un représentant de la cellule nationale : le responsable flux. Ceci conduit les ergonomes à orienter leurs propositions principalement sur la gestion des flux. A cette occasion, une réduction de l'exposition des salariés aux facteurs de risques de TMS est recherchée. Les obstacles à l'intervention identifiés par les intervenants, se situent du côté des marges de manœuvre économiques, c'est-à-dire l'investissement financier dans des améliorations. Les ergonomes doivent tenir compte de ce facteur dans les propositions proposées. De plus, une partie des recommandations doivent être validées par la cellule nationale, qu'il n'a pas été possible de rencontrer même avec l'appui du responsable de région.

Dans l'entreprise B, l'intervention (Landry \& Tran Van, 2010, op. cit.) vise à intégrer la prévention des TMS dès la conception du produit qui sera mis en place dans l'usine. Une équipe prévention pilotée par le médecin du travail cherche à développer un discours clair dans l'entreprise autour de la problématique TMS pour ensuite mettre en place une démarche permettant de cibler les postes vis-à-vis de leur exposition aux facteurs de risques TMS. La place de l'équipe d'ergonomes est alors d'apporter un retour d'expérience sur l'organisation de la prévention dans d'autres entreprises et d'aider l'équipe prévention à construire un référentiel commun sur les TMS. Pour le construire, un modèle des TMS est choisi et discuté en groupe projet. Ce groupe projet est constitué d'un médecin du travail national pilote du projet, de 2 médecins du travail de sites, de 3 infirmières et de 2 ergonomes internes. Le but est de communiquer sur la problématique TMS puis d'identifier les situations de travail prioritaires en matière de prévention. L'objectif est que la démarche soit utilisable par tous les relais santé au travail de l'entreprise sur la problématique TMS et la conduite de projet. Les observations de situations de travail réalisées par les intervenants sont utilisées pour illustrer les modélisations. L'apport de connaissances se situent également sur la présentation des résultats du projet prévention durable des TMS (Caroly et al., 2007, op. cit.) et la discussion sur l'usage des enquêtes épidémiologiques.

Dans ce cas, le principal levier à l'intervention identifé par l'ergonomie relève de la motivation de l'équipe de prévention pour organiser et piloter les actions depuis le siège de l'entreprise. L'intervention est facilitée par la présence d'ergonomes internes qui maintiennent temporellement dans l'entreprise des ressources en ergonomie. A travers les structures syndicales de l'entreprise, le projet de prévention reçoit un support des salariés. La direction est également partie prenante de l'action menée par le service prévention et donne les moyens structurels et organisationnels pour mener le projet. Les obstacles à l'intervention se situent du côté des volumes de production en diminution. Le maintien des commandes, le maintien des objectifs de production sont les principales inquiétudes des responsables production et méthodes au détriment d'un intérêt pour les questions de prévention des facteurs de risques de TMS.

Dans l'entreprise $C$, l'intervention vise à intégrer la prévention des TMS dès la conception de 2 postes de travail. La demande est portée par un ingénieur chef de projet. Ce dernier demande d'abord à l'ergonome d'intégrer au cahier des charges, qui sera diffusé pour appel d'offres à destination des bureaux d'études externes consultés, des éléments qui puissent dans un second temps permettre au groupe projet d'évaluer dans quelle mesure les solutions proposées ont pris en compte la prévention des TMS. Le groupe projet est constitué d'un ingénieur chef de projet, du responsable du service outillage (conception des postes et moyens de travail), du responsable du bureau d'étude produit, du chef d'atelier, d'un responsable achats et d'un responsable qualité. Plusieurs revues de projet ont été réalisées avec le chef de projet et afin d'une part d'argumenter en quoi le seul respect des normes européennes ergonomiques ne suffit pas pour prévenir activement les TMS et d'autre part de disposer d'un référentiel et de définitions communs, une modélisation des TMS a été proposée par l'ergonome. Une fois ce référentiel commun constitué, et pour intégrer au cahier des charges des éléments fonctionnels utiles pour les concepteurs, des analyses ergonomiques de l'activité ont été réalisées sur trois postes de travail dont les tâches se rapprochaient le plus des tâches futures à 
réaliser.

Parmi les leviers favorables à l'intervention, l'ergonome a identifié d'une part l'implication du chef de projet, ce dernier sollicitant régulièrement l'ergonome, cela de manière formelle (réunions pour relecture du cahier des charges, évaluation des solutions) ou de manière informelle (appels téléphoniques, discussions au cours des pauses) et dès les phases initiales du projet. D'autre part, les opérateurs et la hiérarchie de proximité se montrent disponibles, parlent facilement du travail actuel et futur, des aspects positifs comme des difficultés rencontrées.

Cependant, dès les premiers échanges relatifs à ce projet, deux obstacles à l'intervention sont identifiés :

- les dirigeants, identifiant que l'entreprise perd peu à peu son leadership et sa vitrine technologique, imposent de concevoir des postes innovants (d'un point de vue technique, consommation d'énergie, process de fabrication) : l'écart important entre les analyses réalisées sur des postes conçus au début des années 80 et utilisés comme situation de référence et le travail futur possible étant identifié comme conséquent, le risque d'erreur de conception s'en trouvait augmenté.

- Les marges de manœuvres financières sont rapidement identifiées comme faibles, et le budget alloué au projet se présente trop serré dès les premières évaluations de solutions. Quelques mois après le début des premières études, le budget est revu à la baisse, ce qui contraint le groupe projet à faire des choix techniques qui desserviront la qualité de vie au travail (systèmes d'aide à la manutention manuels plutôt qu'électriques, par exemple).

Un troisième obstacle accompagnera l'intervention : le gel du projet à deux reprises en deux ans, avec à chaque reprise des changements d'acteurs dans l'équipe projet, ceci rendant difficile l'investissement dans le travail des acteurs internes comme des prestataires et diluant la mémoire de projet.

La comparaison inter-cas peut mettre en évidence des influences des caractéristiques des interventions sur les effets produits (Baril-Gingras et al., 2006, op. cit.) :

- position de l'intervenant (interne/externe),

- mise en place d'une démarche participative,

- le support budgétaire,

- l'implication des décideurs.

Dans les cas d'interventions A et B, l'intervenant est externe à l'entreprise, ceci a pu être identifié comme un obstacle à l'intervention (Baril-Gingras et al., 2006, op. cit.) car la ressource temporelle de l'ergonome est moindre dans les cas où l'intervenant est externe à l'entreprise. Cependant, le cas d'intervention B rejoint le cas C, puisque l'ergonome externe peut s'appuyer sur des ressources internes en ergonomie et en prévention.

Les 3 cas d'interventions s'appuient sur une démarche participative reposant sur des structures projets telles que groupe de pilotage, groupe de travail et comité de suivi. Ces structures sont des facteurs favorables à la production d'effets de l'intervention et à la pérennisation de la démarche au-delà de la présence des ergonomes.

Dans les cas A et C, les marges manœuvres budgétaires sont faibles, ce qui a un effet sur les caractéristiques de l'intervention : les ergonomes recherchent plutôt des solutions demandant un faible investissement financier. Dans l'intervention $\mathrm{B}$, le projet vise à mobiliser des ressources internes (service prévention, service production et service méthode), il n'a pas non plus de support financier massif.

Dans le cas A et le cas C, la direction, les décideurs principaux ne sont pas directement impliqués dans une structure projet, bien que la démarche s'appuie sur des représentants de décideurs, ceci peut être un obstacle aux effets de l'intervention dans la mesure où Baril-Gingras, Bellemare et Brun 
(2006, op. cit.) ont identifié que « les responsables qui sont absents lors de la recherche de solutions vont plutôt exprimer un certain scepticisme vis-à-vis de l'intervention ».

Les leviers et obstacles identifiés par les ergonomes sont répartis parmi les 3 cas d'intervention (Tableau 4).

\begin{tabular}{|c|c|c|c|c|}
\cline { 2 - 5 } \multicolumn{1}{c|}{} & $\begin{array}{c}\text { Position de } \\
\text { l'intervenant interne }\end{array}$ & $\begin{array}{c}\text { Mise en place démarche } \\
\text { participative }\end{array}$ & Support budgétaire & $\begin{array}{c}\text { Implication des } \\
\text { décideurs }\end{array}$ \\
\hline A & & $\mathrm{x}$ & & \\
\hline B & & $\mathrm{x}$ & & $\mathrm{x}$ \\
\hline C & $\mathrm{x}$ & $\mathrm{x}$ & & \\
\hline
\end{tabular}

Tableau 4 : Répartition des leviers à l'intervention selon les 3 cas.

Table 4: Summary of levers used in the three projects.

Dans chacun des cas les ergonomes ont pu saisir des opportunités en s'appuyant sur les leviers (Landry, 2008, op. cit.) pour tenter de minimiser les impacts des obstacles identifiés. Ainsi, la comparaison inter-cas (Leplat, 2002, op. cit.) permettra de rapporter les effets produits par l'intervention compte tenu des caractéristiques des intervenants, de l'intervention et des effets de contexte dans lequel l'intervention se déroule. Les influences des caractéristiques de l'intervention, des obstacles et leviers identifiés sur les effets observés pour un cas pourront être contrebalancés par les effets produits pour les deux autres cas. La répétition inter-cas des effets produits par l'intervention cherche à isoler les effets de ces variables (Yin, 1994, op. cit.) par répétition artificielle (Falzon, 1996, op. cit.).

Nous venons de caractériser d'une part le contexte d'intervention et l'intervention réalisée. La comparaison inter-cas permettant de contrebalancer les effets de contexte ou de caractéristiques d'intervention sur l'usage d'un modèle des TMS comme référentiel commun. Nous allons maintenant préciser comment les effets de ce modèle vont être caractérisés, c'est-à-dire quel cadre théorique permettra de déterminer si une caractéristique de l'intervention (le modèle des TMS) a produit des effets sur les représentations des acteurs de l'entreprise.

\section{4.- Caractérisation des effets produits par l'utilisation d'un modèle des TMS comme référentiel commun}

Afin de caractériser les effets produits par l'utilisation d'un modèle des TMS comme référentiel commun, il est utile de préciser le cadre théorique qui a servi pour la construction d'indices de réussite. C'est-à-dire que d'après le cadre théorique suivant, on pourra déterminer si le choix d'un modèle des TMS comme référentiel commun entre ergonomes et intervenants a permis d'agir sur les représentations des acteurs de l'entreprise comme le propose la figure 1.

Pour Daniellou (1999) ou Leplat (1992), si l'usage d'un modèle commun est utile pour guider et structurer l'action ergonomique, le suivi d'un modèle ne garantit pas la qualité et la réussite de l'intervention. La pertinence d'un modèle comme objet intermédiaire (Jeantet, 1998, op. cit.) dans une situation se vérifie si le modèle présente différentes qualités : il doit se montrer opérant, communiquant, malléable (Vinck, 2000).

- Selon Wisner (in Durrafourg \& Vuillon, 2004) un modèle opérant vue comme objet intermédiaire, est un modèle qui permet de créer des repères de connaissances sur un problème entre acteurs qui doivent ensuite résoudre le problème.

- Un modèle communiquant, vu comme objet intermédiaire, permet d'établir des liens entre des acteurs, participe à la création de réseaux sociaux autour de la résolution de problème.

- Enfin, un modèle malléable, vu comme objet intermédiaire, est un modèle qui est transitoire, qui peut être changé, amélioré au fur et à mesure de l'avancée du projet. 
Pour mener cette recherche nous avons déterminé d'après la théorie de Vinck (2000, op . cit.) sur les qualités des objets intermédiaires, des indices d'effets :

Si le modèle utilisé permet à chacun de connaître le phénomène TMS, et donc de participer à un projet de prévention où des pistes de prévention sont recherchées ; de même si ce modèle doit permettre de créer des liens entre des acteurs de l'entreprise qui n'ont pas l'habitude de travailler ensemble (service des méthodes et service des ressources humaines, ou encore bureau d'étude et service de prévention); et enfin, si le modèle est suffisamment malléable pour l'adapter au contexte des interventions, et permettre aux acteurs des projets de proposer des améliorations pour en augmenter les qualités opératives, alors nous pourrons observer les points suivants dans chacun des cas (indices) :

L'utilisation par les acteurs de l'entreprise du vocabulaire et des concepts présentés dans le modèle à l'occasion d'échanges sur les projets (Saint-Arnaud, 1992). Cela témoigne de la compréhension de la problématique TMS par les acteurs et d'un partage de ce référentiel parmi des acteurs aux missions différentes.

L'identification par chaque acteur de pistes de prévention à l'occasion de groupe projet constitués pour l'intervention et faisant travailler ensemble des acteurs qui n'échangent pas habituellement. Ce qui constitue pour notre objectif de recherche, des indicateurs de la structuration d'une dynamique de prévention dans l'entreprise.

Des propositions d'amélioration du modèle par les acteurs des entreprises. Ceci témoignant de l'appropriation du modèle pour structurer la prévention et la faire perdurer dans le temps.

\section{2.- Choix d'un modèle de TMS comme support au référentiel commun}

Ainsi, dans chacun des cas d'interventions, les ergonomes ont recherché dans la littérature des modèles de TMS permettant de construire l'intervention et servant de référentiel commun sur la problématique. Pour cela, une revue bibliographique a été effectuée et un modèle a été choisi parmi ceux trouvés et largement utilisés dans les interventions ergonomiques francophones.

\section{1.- Revue bibliographique des différents modèles de TMS}

Face à la demande formulée aux intervenants en 2008, les ergonomes ont effectué une rapide revue bibliographique principalement dans des ouvrages ou rapports de recherche en ergonomie. Ils ont ainsi trouvé les modèles suivants (non exhaustifs de l'étendue des modèles scientifiques produits sur les TMS) les plus fréquemment utilisés : Franchi, 1997 (modèle A, Figure 2) ; Bourgeois, Lemarchand, Hubault, Brun, Polin et Faucheux, 2000 (modèle B, Figure 2) ; Nahon \& Arnaud, 2001 (modèle C, Figure 2) ; Bellemare, Marier, Montreuil, Allard et Prévost, 2002 (Figure 3), et de Denis, St Vincent, Jetté, Nastasia et Imbeau, 2005 (modèle D, Figure 2).

Chacun des modèles recensés, présentent des aspects différents des TMS et de l'action de prévention. Ces modélisations sont complémentaires et non pas concurrentes, d'autant plus qu'elles correspondent à des temporalités différentes en termes d'avancée de connaissances sur l'étiologie des TMS et l'organisation des démarches de prévention. Cependant, comme nous le verrons dans la figure 2, les modélisations représentent graphiquement plutôt l'étiologie des TMS pour certain ou plutôt la démarche d'intervention pour d'autres. 


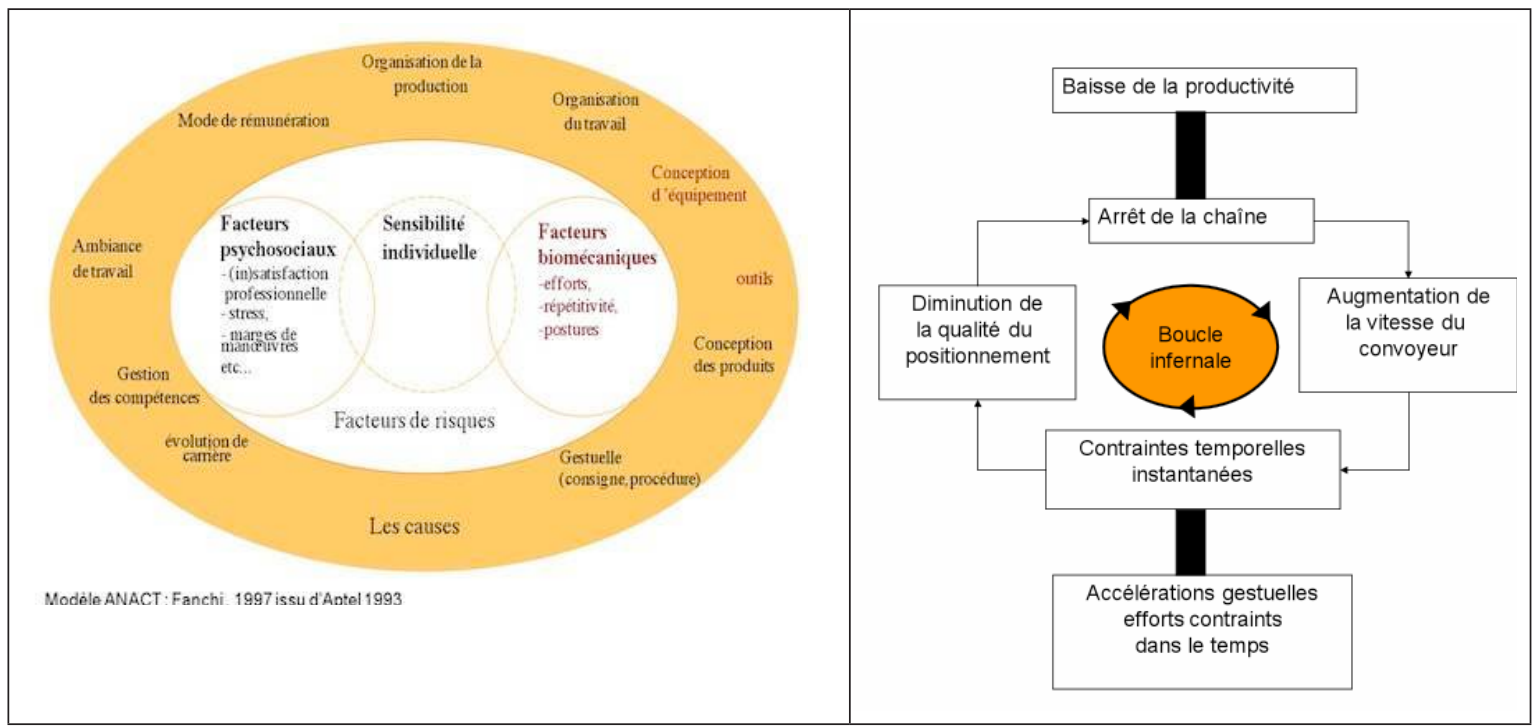

Modèle A : Fanchi 1997 d'après Aptel, 1993

Modèle C : Nahon et al., 2001

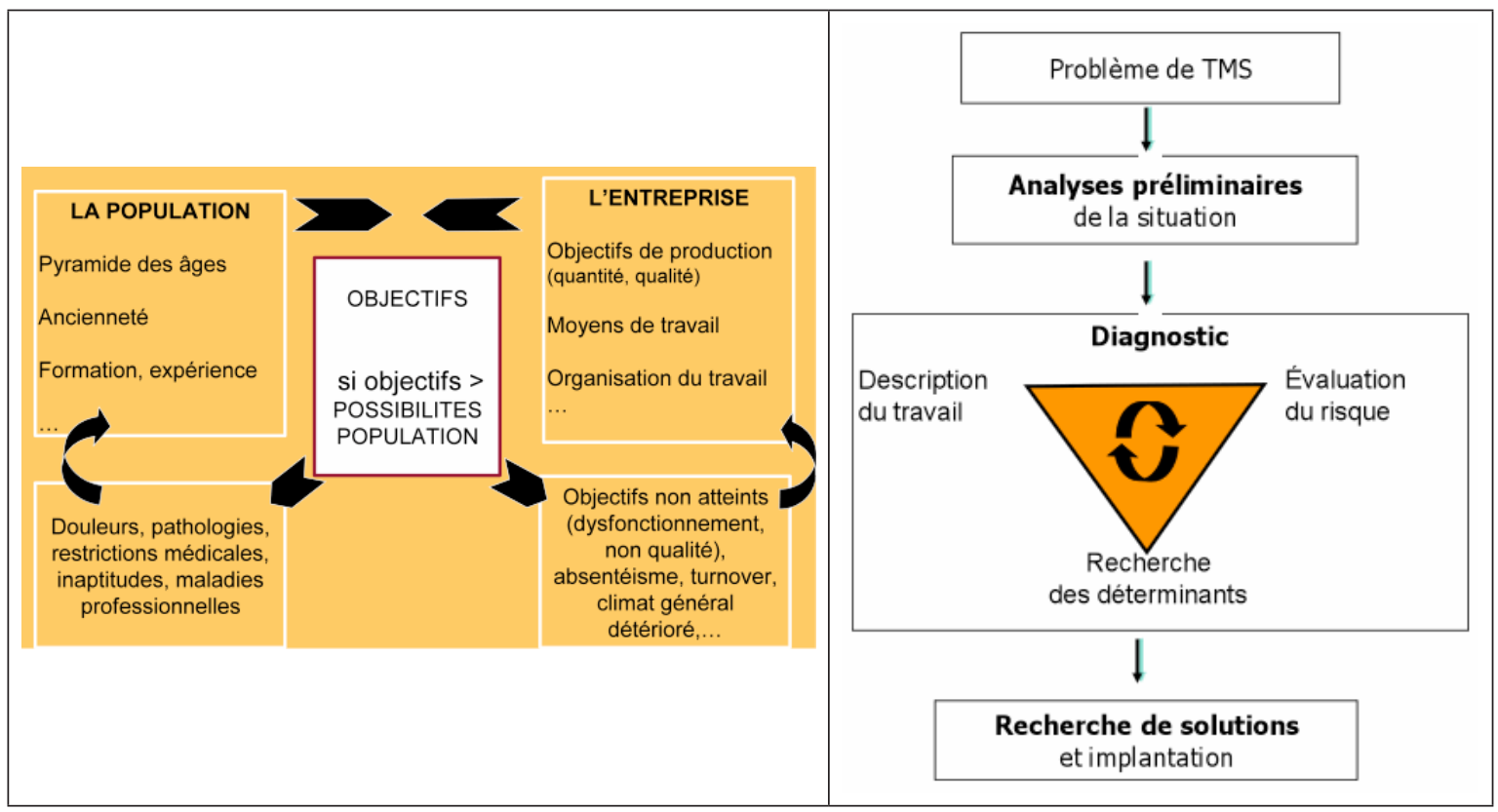

Modèle B : Bourgeois et al., 2000

Modèle D : Denis et al., 2005

Figure 2 : Modèles étudiés pour servir de référentiel commun.

Figure 2: Models studied to supply a standard set of references.

Le modèle de Franchi (1997, op. cit.), utilisé dans le réseau ANACT tiré des travaux d'Aptel (1993) ,place les facteurs de risques de TMS : facteurs psychosociaux, sensibilité individuelle, facteurs biomécaniques au centre d'un réseau de causes organisationnelles et productives (modèle A, Figure 2). Le modèle de Bourgeois, Lemarchand, Hubault, Brun, Polin et Faucheux (2000, op. cit.) est un modèle dynamique qui pointe les origines des TMS, les conséquences, et les moyens de diminuer les conséquences néfastes. Il présente les TMS comme un effet du dépassement des capacités de la population de salariés par les objectifs donnés (modèle B, Figure 2)

Le modèle de Nahon et Arnaud (2001, op. cit.) se centre sur la situation de travail et la notion de marges de manœuvre. Il montre comment les analyses du travail peuvent mettre à jour des boucles infernales expliquant l'exposition des salariés aux facteurs biomécaniques et rigidifiant les marges 
de manœuvre des salariés à l'origine des troubles (modèle C, Figure 2). Dans ces trois modèles, la démarche ergonomique n'est pas visuellement représentée, elle repose sur les explications données par les intervenants et l'usage d'autres modèles d'action.

Le modèle de Denis, St Vincent, Jetté, Nastasia et Imbeau (2005, op. cit.) présente comment d'un problème de TMS la recherche de pistes de prévention s'appuie sur une phase de diagnostic visant à décrire le travail pour évaluer le risque TMS et trouver les déterminants aux facteurs de risque (modèle D, Figure 2). Dans ce modèle l'étiologie des TMS n'est pas représentée (pas de spécifications des facteurs de risque, des manifestations des TMS ou encore de déterminants aux facteurs de risques). Enfin le modèle de Bellemare, Marier, Montreuil, Allard et Prévost (2002, op . cit.) représente sur la même schématisation (Figure 3) les facteurs de risques TMS et leurs déterminants mais aussi l'organisation d'une action de prévention. Compte tenu des demandes formulées, il est apparu important aux intervenants de disposer d'un modèle expliquant l'étiologie des TMS, mais également les grandes phases d'organisation d'une action de prévention. C'est pour cette caractéristique que les intervenants ont choisi le modèle de Bellemare, Marier, Montreuil, Allard et Prévost (2002, op. cit.).

\section{2.- Présentation du modèle de Bellemare, Marier, Montreuil, Allard et Prévost (2002, op. cit.)}

Dans le modèle de Bellemare, Marier, Montreuil, Allard et Prévost (2002, op. cit.), la partie étiologie des TMS est située sur la droite du schéma et considère les TMS comme des effets sur l'opérateur produits par une sur-exposition de l'opérateur à des facteurs de risque TMS. Ces facteurs de risques sont présentés comme résultant des caractéristiques du travail appelées « déterminants ». Ces déterminants sont classés en 3 familles :

- les déterminants techniques (outils, matières premières...)

- les déterminants organisationnels (cycles de travail, rotation...)

- les déterminants humains (compétences, collectif...)

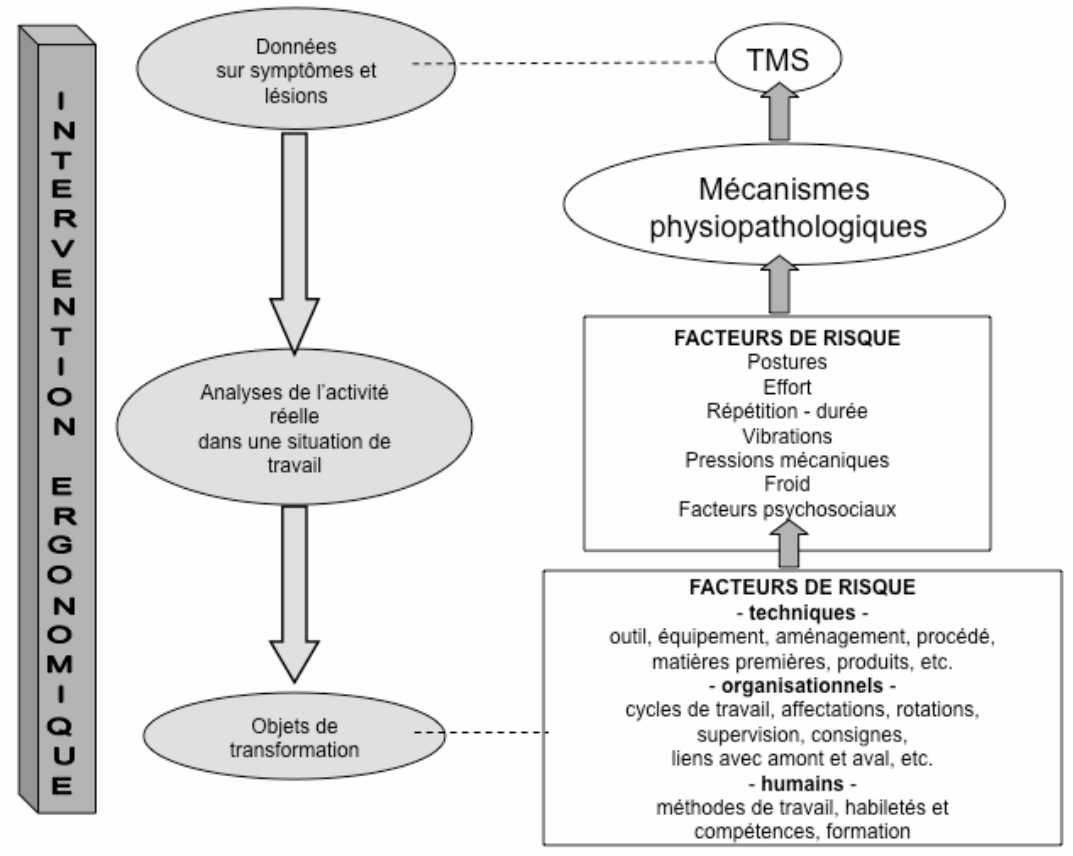

Figure 3 : Modèle des TMS de Bellemare et al. (2002)

Figure 3: The MSD model used by Bellemare et al.(2002) 
Dans ce modèle, la démarche de prévention, ici l'intervention en ergonomie, est située sur la gauche du schéma. Le schéma indique que l'ergonome tout au long de son intervention, cherche à relier les symptômes et lésions affectant les opérateurs aux déterminants des situations de travail. Pour cela l'ergonome utilise divers outils notamment l'analyse du travail.

Ainsi ce schéma illustre qu'un facteur de risque peut être relié à une chaîne de déterminants. Ceci peut s'avérer utile pour montrer l'importance de créer des liens entre les acteurs de l'entreprise. En mettant en lien les facteurs de risque avec les déterminants du travail (comme le font également d'autres modèles présentés dans cet article) la modélisation montre l'importance d'axer la recherche de solutions sur les déterminants du travail comme cible dans la prévention des TMS. Il est alors plus facile aux différents acteurs de l'entreprise de faire des liens entre leurs projets (production, organisation du travail...) et les conséquences de ces projets sur les opérateurs.

Par exemple, la recherche de solutions pour réduire des facteurs biomécaniques ne permet pas de trouver autant de pistes de recommandations que la réflexion sur les outils, les cycles de travail, le procédé de fabrication, la formation des opérateurs, les liens entre les postes amonts et les postes avals.

Ce modèle a donc servi de support à la construction du référentiel commun que les intervenants souhaitaient partager à l'occasion des 3 cas d'intervention présentés précédemment.

\section{3.- Illustration d'effets produits par l'usage du modèle sur les acteurs de l'entreprise}

Dans les trois cas d'intervention, les ergonomes ont pu caractériser des manifestations de la construction d'un référentiel commun à travers l'utilisation d'un modèle de TMS en référence aux qualités des objets intermédiaires (verbalisation, analyse de contenu de documents, observations du déroulement du projet). Ces effets sont repris dans le tableau 5 pour chaque cas.

\begin{tabular}{|c|c|}
\hline 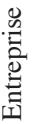 & $\begin{array}{l}\text { Effets produits sur les représentations des acteurs de l'entreprise attribuables à l'usage d'un modèle de TMS vu comme objet } \\
\text { intermédiaire }\end{array}$ \\
\hline A & $\begin{array}{l}\text { Réutilisation des concepts (déterminants, facteurs de risque) dans les échanges en groupe projet quelque soit le niveau } \\
\text { hiérarchique. } \\
\text { Recherches de solutions en groupe projet et groupe de travail sur les différents déterminants. } \\
\text { Propositions d'améliorations du modèle pour intégrer les particularités de l'entreprise en groupe projet. } \\
\text { Sensibilisation à la problématique TMS des autres directeurs de magasin de la région. }\end{array}$ \\
\hline B & $\begin{array}{l}\text { Réutilisation des concepts (déterminants, facteurs de risque) dans les échanges verbaux entre médecins du travail, infirmières } \\
\text { puis avec les ingénieurs méthodes et production, } \\
\text { Propositions d'améliorations du modèle, développement du nouveau modèle pour sensibiliser les ingénieurs méthodes et } \\
\text { production à la problématique TMS. } \\
\text { Recherches de secteurs prioritaires en structurant un groupe projet prévention-production sur la base de la réalisation de } \\
\text { diagnostics des situations de travail à l'aide du modèle explicatif produit. }\end{array}$ \\
\hline $\mathrm{C}$ & $\begin{array}{l}\text { Réutilisation et diffusion des concepts parmi les acteurs de l'entreprise } \\
\text { Evolution du regard sur les stratégies opératoires de l'Homme au travail } \\
\text { Evolution du regard sur les apports de l'ergonome dans les projets de conception (pas uniquement un garant du respect des } \\
\text { normes anthropométriques) }\end{array}$ \\
\hline
\end{tabular}

Tableau 5 : Synthèse des effets produits sur les représentations des acteurs de l'entreprise, attribuable à l'usage d'un modèle de TMS vu comme objet intermédiaire

Table 5 : Synthesis of the effects produced on the companies' actors and attributable to the use of a MSD model considered as an intermediary object 


\section{1.- Comparaison inter-cas}

Dans ces trois situations, indépendamment des effets de contexte ou de structuration de l'intervention, le modèle a permis de mobiliser les acteurs de l'entreprise et d'augmenter la prise en charge collective des TMS :

- L'usage du modèle comme référentiel commun a permis de rendre compréhensible pour les non-spécialistes de la santé au travail ou de la prévention des risques professionnels, la logique suivie pour expliquer la survenue des TMS, l'organisation de la démarche de prévention, et les données à récupérer (verbalisation des acteurs des entreprises mobilisées dans l'intervention).

- La représentation graphique facilite la compréhension du problème (les TMS) et de la démarche d'intervention en ergonomie. Le modèle rencontre la qualité « communicante » des objets intermédiaires (verbalisation des acteurs des entreprises mobilisées dans l'intervention).

- La problématique TMS est identifiée, et des pistes pour permettre le déroulement de l'intervention en ergonomie et en prévention sont proposées (verbalisation des acteurs des entreprises mobilisées dans l'intervention).

- Le référentiel commun est basée sur une évolution d'un niveau micro au poste (facteurs de risque pour la santé) à un niveau plus macro (déterminants de la situation de travail).

- Les acteurs de l'entreprise comprennent qu'une prévention efficace repose sur une remise en question des déterminants du travail, et non sur « des mauvaises pratiques » des opérateurs.

- Les acteurs de l'entreprise s'axent sur la recherche des déterminants pathogènes des situations de travail.

Nous nous proposons d'illustrer chacun des 3 indicateurs présentés au chapitre 1.4) (caractère opérant, caractère communicant et caractère malléable) en prenant 2 cas.

\subsection{1.- Exemple d'effets de compréhension de la problématique (effet du caractère opérant du modèle)}

Selon Vinck (2000, op . cit.) pour assurer la qualité de référentiel commun, un modèle doit permettre de créer des repères de connaissances sur un problème entre acteurs qui doivent ensuite résoudre le problème.

Dans l'intervention réalisée dans l'entreprise $\mathrm{C}$, le modèle a été présenté afin d'argumenter l'idée selon laquelle le simple respect des normes ergonomiques de conception ne suffirait pas à prévenir le risque TMS, et qu'il serait utile d'intégrer des repères fonctionnels au cahier des charges (repère de type : lorsque l'opérateur est au pupitre de commande, et dirige le pont roulant pour disposer le produit dans le bâti, il est nécessaire qu'il ait une vision directe et optimale sur la zone $\mathrm{Z}$ du produit, qui est la plus fragile et risque de heurter le bâti). Les débats qui ont suivi la présentation du modèle ont permis à l'intervenant d'identifier une évolution à deux niveaux du point de vue des acteurs du groupe projet : d'abord une évolution relative aux stratégies opératoires de l'Homme au travail. La présentation du modèle, illustrée d'exemples tirés des analyses de terrain, a permis de rappeler les concepts de variabilité produit, de variabilité inter et intra-individuelle, pour ainsi argumenter l'intérêt de prévoir des espaces qui permettent aux opérateurs, tout en respectant les énormes contraintes de la construction aéronautique, de se construire des marges de manœuvre. Ensuite, une évolution des points de vue relative à la place et à l'intérêt de l'analyse ergonomique du travail dans les phases initiales d'un projet de conception. D'un garant du respect de l'intégration des normes européennes ergonomiques, l'ergonome est devenu un acteur de la conception, d'une part autorisé à intégrer des repères fonctionnels, et d'autre part en phases d'évaluation des solutions, interrogé sur l'adéquation entre les solutions proposées par les concepteurs et l'activité de travail observée en situations de référence.

Cette évolution du regard des acteurs du groupe s'est confirmée lorsque dans d'autres projets ultérieurs au projet présenté, l'ergonome a été sollicité dès les phases de préparation du cahier des 
charges fonctionnel, et impliqué aux différentes phases des projets. En ce sens, nous argumentons l'idée selon laquelle la présentation du modèle de Bellemare, Marier, Montreuil, Allard et Prevost (2002, op . cit.) au groupe projet a contribué à construire entre les acteurs des repères de connaissances sur le problème de conception, et ainsi à remplir sa fonction de modèle opérant.

\subsection{2.- Exemple de proposition d'améliorations du modèle par les acteurs de l'entreprise (effet du caractère communiquant et malléable du modèle)}

Selon Vinck (2000, op . cit.) pour rencontrer les caractéristiques des objets intermédiaires, un modèle doit être malléable, c'est-à-dire permettre aux acteurs de l'entreprise une appropriation.

Dans l'entreprise A, le modèle des TMS de Bellemare et al. (2002, op . cit.) (Figure 3) a été présenté au groupe projet (composé du directeur de magasin et des responsables de secteur) lors de la réunion de lancement. Le but étant d'expliquer comment l'intervention allait se dérouler et sur quels points allaient porter le diagnostic. Puis, ce modèle a servi de support à la présentation du pré-diagnostic des ergonomes. Ce même pré-diagnostic a été présenté en comité de suivi avec le directeur de région.

En débriefing avec le directeur de magasin, nous convenons de faire travailler le groupe projet sur ce sujet. Les acteurs du groupe ont alors décidé d'adapter le modèle de Bellemare et al. (2002, op . cit.) comme support à la formation des directeurs de magasin (Figure 4).

En s'appuyant sur les éléments transmis dans le pré-diagnostic, les responsables de secteur et le directeur du magasin aidés des ergonomes, ont modifié le modèle pour mieux rendre compte du travail effectué par les opérateurs du magasin.

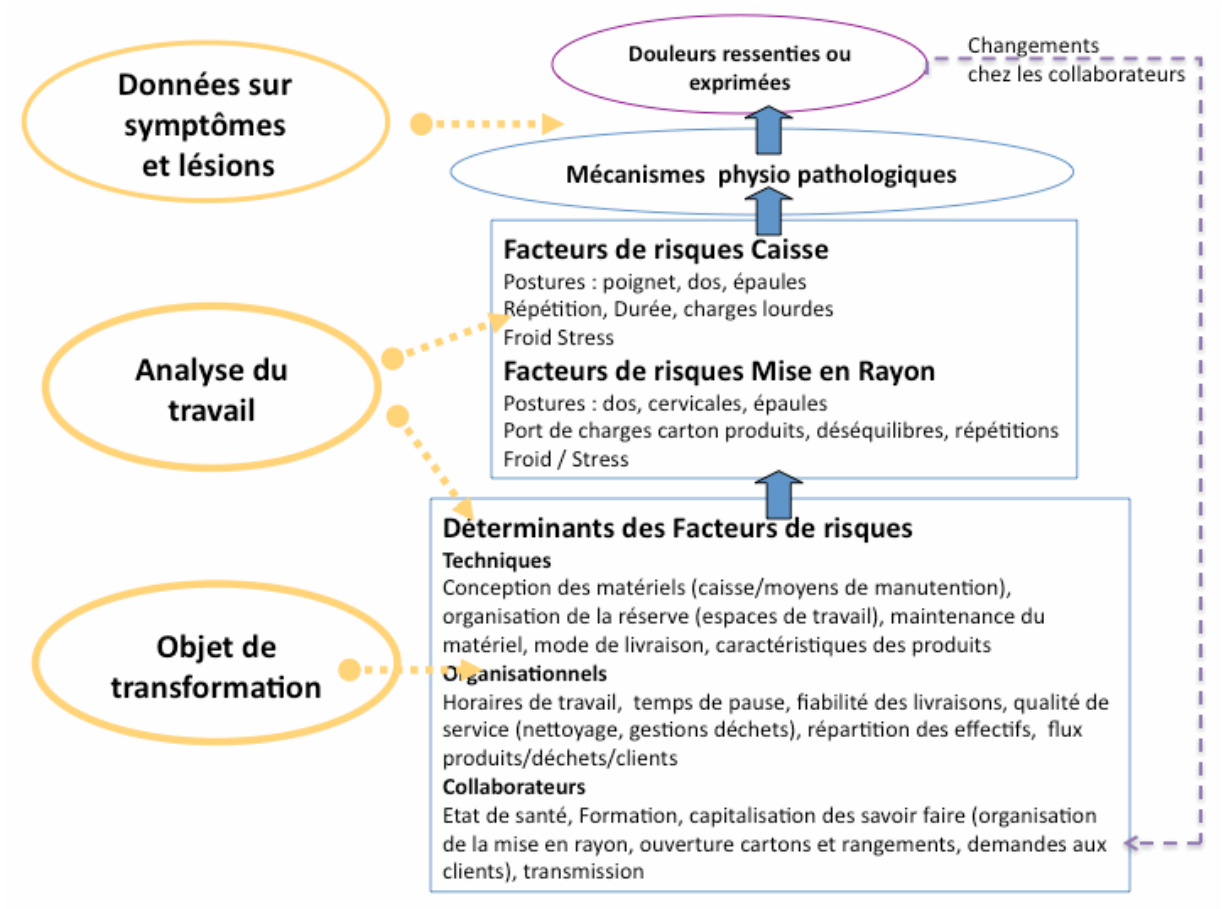

Figure 4 : Modèle des TMS adapté par les participants au groupe projet

Figure 4: MSD model adapted by members of the project group

Partant du constat qu'aucun opérateur du magasin n'avait jusqu'à présent déclaré de TMS, les participants au groupe ont proposé de remplacer «TMS » par douleurs ressenties ou exprimées. De même, ils ont jugé plus pertinent de détailler les facteurs de risques pour 2 catégories d'activités : le travail en caisse et le travail de mise en rayons. Ils ont souhaité détailler les localisations corporelles qui étaient particulièrement exposées à travers ses activités (postures) et ajouté des éléments sur les manutentions. Du côté des déterminants des facteurs de risques, en utilisant le pré-diagnostic, ils ont 
spécifié les déterminants (voir Figure 4).

Les membres du groupe ont trouvé important d'intégrer une flèche de rétroaction, c'est-à-dire d'insister sur le fait que l'opérateur permet d'atteindre les objectifs et que cette performance repose sur une diminution des douleurs. Ceci constituant pour eux un moyen de convaincre les directeurs de magasins d'engager des démarches de prévention. Ils ont volontairement choisi dans les déterminants d'être très concrets pour permettre aux directeurs de déceler des solutions de prévention.

Cette proposition faite par le groupe projet a été utilisée en restitution finale à tous les directeurs de magasins de la région (pour plus de détails sur les effets produits voir Landry \& Feillou, 2008, op. cit.). Ceci est une illustration du caractère malléable et communiquant du modèle de Bellemare et al. (2002, op. cit.).

Ces deux illustrations montrent comment le modèle de Bellemare et al. (2002, op . cit.), positionné dans l'intervention comme un moyen de construire un référentiel commun sur les TMS, a engendré des effets sur les acteurs de l'entreprise : ceux-ci ont acquis un certain nombre de connaissances sur une problématique de santé au travail, ont utilisé le modèle pour échanger entre eux et ont proposé des adaptations afin que celui-ci corresponde à leur réalité et à leurs besoins d'utilisation. Bien que les illustrations choisies ciblent sur une caractéristique plutôt qu'une autre, elles montrent comment les 3 caractéristiques des objets intermédiaires sont imbriquées les unes dans les autres (un modèle n'est malléable, que s'il est opérant et communicant, un modèle est opérant s'il est communicant et malléable...). Cette imbrication pouvant reposer sur un effort stratégique de la part des ergonomes : par exemple, pour augmenter le caractère opérant du modèle, on l'illustre avec des situations de l'entreprise, on s'appuie alors sur le caractère malléable du modèle.

\section{4.- Proposition d'un modèle opérant pour la prévention des TMS}

\section{1.- Incrémentation du modèle suite à son utilisation en intervention}

L'évolution des connaissances scientifiques relatives aux TMS, mais aussi l'utilisation de ce modèle à l'occasion d'interventions de prévention a conduit les utilisateurs à proposer des améliorations correspondant à un meilleur couplage modèle/réalité observée (Leplat, 1992, op. cit.). C'est-à-dire que l'expérience vécue des TMS par les acteurs de l'entreprise, y compris les ergonomes, les a conduit à apporter six types d'amélioration au modèle de Bellemare et al. (2002, op. cit.) dans le but de maximiser ses qualités intrinsèques.

Ainsi, nous avons caractérisé avec plus de précisions :

1. les facteurs de risque TMS, divisés en :

- facteurs biomécaniques (que l'on peut identifier selon l'intensité, la fréquence, la durée,

l'amplitude, la vitesse, la précision et l'asymétrie des postures et gestes),

- facteurs mécaniques (pressions mécaniques, chocs, vibrations),

- facteurs d'ambiance physique (froid, bruit),

- facteurs psychosociaux (soutien social, pouvoir d'agir, demande psychologique),

- facteurs individuels (âge, sexe, diabète, hypothyroïdie).

2. Nous avons ensuite cherché à préciser la notion de TMS qui peut, en fonction des interlocuteurs, être représentative de différentes réalités et par conséquent être à l'origine d'incompréhensions. Pour cela nous avons décliné tout ce qui relève des effets, « désagréments » de santé (Volkoff, 2005) subis par l'opérateur.

En effet, avant le stade de déclaration de la maladie professionnelle appelée TMS (définie dans le tableau 57 des maladies professionnelles par « une affection péri-articulaire provoquée par certains gestes et postures au travail »), l'opérateur évoquera des plaintes, des douleurs, des lésions, des in- 
capacités... qui sont autant d'indices susceptibles d'alerter de la nécessité d'évaluer plus finement l'ampleur du problème, voir d'impulser une démarche de prévention des TMS. D'autant plus que l'usage de mots comme «maladies professionnelles » ou « accidents du travail » peuvent par ailleurs nuire à la mobilisation des acteurs de l'entreprise pour la prévention (Caroly et al., 2007, op. cit.).

3. Nous avons ensuite intégré l'idée [co-construite par les acteurs de l'entreprise A et les intervenants (Landry \& Feillou, 2008)] selon laquelle les désagréments subis par l'opérateur influent de manière rétroactive sur les déterminants du travail, rigidifiant la situation de travail : par exemple, pour causes de douleurs, un opérateur qui ne peut plus utiliser certains outils ou équipements, tenir certains postes de travail, respecter les temps de cycle, etc. sera susceptible de compliquer l'organisation prescrite du travail ou de fragiliser le collectif de travail. Le système de travail risquerait alors d'entrer dans une « boucle infernale» (Daniellou, 1998) et les solutions de prévention seraient plus lourdes et complexes à mettre en œuvre.

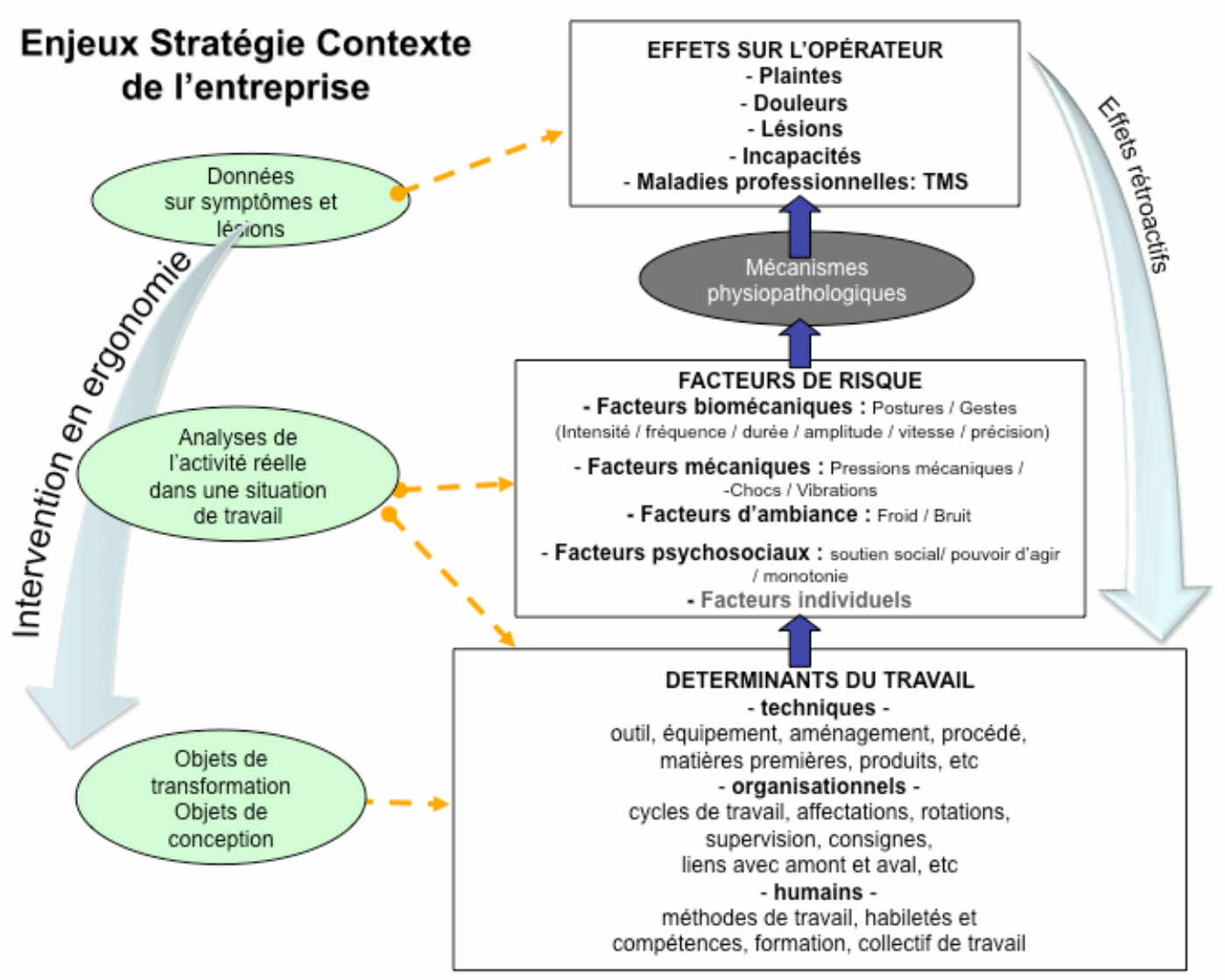

Figure 5 : Proposition d'un modèle opérant de prévention des TMS

\section{Figure 5 : Suggesting for an operating model for MSD prevention intervention}

4. Dans le cadre intitulé initialement « déterminants », en accord avec les travaux de Vézina (2001), ou encore Coutarel (2004), nous avons remplacé le terme « déterminants » par « déterminants du travail », mais que l'on peut aussi définir par « déterminants des facteurs de risque » (Coutarel, Vézina, Berthelette, Aublet-Cuvelier, Descatha, Chassaing et al., 2009). Toujours dans ce cadre, les travaux de Caroly (2010 à paraître) ont aussi montré les enjeux de l'activité collective pour la santé au travail ; le collectif de travail a donc été intégré comme un déterminant du travail susceptible d'agir sur l'exposition des opérateurs aux facteurs de risque TMS.

5. Selon le modèle initial, chaque déterminant du travail peut devenir un objet de transformation. Nous avons pour notre part utilisé ce modèle dans des projets de conception de situations de travail 
innovantes. L'objectif de l'intervention en ergonomie n'était donc pas d'appréhender les déterminants du travail futur probable comme des objets de transformations, mais bien comme des objets de conception. Pour cela, nous avons intégré au modèle les déterminants du travail vus comme des objets de conception.

6. Enfin, divers retours d'expérience, mais aussi des écrits récents (Aptel \& Vézina, 2008, op. cit. ; Caroly et al., 2007, op. cit. ; Tran-Van, Landry, \& Martin, 2008...) rappellent que pour inscrire durablement la prévention dans le développement des entreprises, et pour correspondre aux réalités de structuration de celles-ci, tant la démarche d'intervention que les cibles de transformation se devront d'être adaptées au contexte et à la stratégie de développement de l'entreprise.

L'efficacité de cette évolution du modèle initial est à tester à travers sa diffusion et son utilisation dans des contextes où les utilisateurs du modèle ne sont pas les concepteurs. En effet, les concepteurs d'un modèle sont peut-être plus à l'aise que les autres utilisateurs pour maximiser les qualités opératives du modèle quel qu'il soit. Par exemple, la diffusion du modèle de Franchi dans le réseau ANACT/ ARACT et son utilisation fréquente par les chargés de mission du réseau, confère à ce modèle des qualités opératives sur la base des dimensions que nous avons mentionnées précédemment (Caroly et al., 2007, op. cit.). De même il serait intéressant d'évaluer quels seraient les effets produits dans d'autres contextes d'interventions ou avec des caractéristiques très différentes des interventions décrites dans cet article.

\section{2.- Conclusions, limites et perspectives}

Daniellou (1999) rappelle qu'un modèle est toujours réducteur, qu'il ne rend pas compte de la complexité de la réalité. Le modèle présenté ci-dessus n'échappe pas à cette règle, et notre choix a donc été orienté vers la recherche du détail juste pour que le modèle soit opérant et compréhensible par les différents acteurs de l'entreprise, et pas seulement les spécialistes de la santé au travail.

Toutefois, et malgré son caractère intrinsèquement réducteur, l'usage satisfaisant de ce modèle dans différents contextes et à plusieurs reprises depuis ces trois cas semble confirmer que cette modélisation remplit les fonctions de ce que de Terssac et Chabaud (1990) définissent comme un référentiel opératif commun, et permet d'atteindre les buts de construction sociale de l'intervention. Nous savons par ailleurs qu'il est utilisé par d'autres ergonomes et nous attendons avec intérêts leurs commentaires sur l'usage de cette modélisation. Cela permettrait de voir les effets produits par ce modèle dans d'autres caractéristiques d'intervention (selon celles décrites dans le tableau 4), notamment en faisant varier les caractéristiques des intervenants, ou le contexte d'intervention. Ces expériences permettraient d'augmenter la puissance de généralisation des résultats observés, puisque ce travail ne s'appuie actuellement que sur trois cas.

L'incrémentation de ce modèle et son utilisation en recherche-action offre également des perspectives de recherche en vue de préciser les concepts de douleurs, de plaintes, qui sont des indicateurs utilisés par les médecins de travail mais peu pris en compte dans les modes de management des entreprises. De même, les liens entre facteurs psychosociaux et TMS sont à préciser plus solidement pour engager des pistes de prévention qui justifient la prise en compte des dimensions organisationnelles et collectives du travail.

\section{RÉFÉRENCES}

Aptel, M. (1993). Quantification des facteurs de risques biomécaniques du syndrome du canal carpien. Actes du $28^{\text {ème }}$ congrès de la SELF, Genève.

Aptel, M., \& Vézina, N. (2008). Quels modèles pour comprendre et prévenir les TMS ? Pour une approche holistique et dynamique. Actes du $2^{\text {ème }}$ congrès francophone sur les TMS, Montréal.

Baril-Gingras, G., Bellemare, M., \& Brun, J.-P. (2004). Intervention externe en santé et en sécurité du travail : un modèle pour comprendre la production de transformations à partir de l'analyse 
d'interventions d'associations sectorielles paritaires. Etudes et recherches, IRSST R-367. http://www. irsst.qc.ca/fr/_publicationirsst_100042.html

Baril-Gingras, G., Bellemare, M., \& Brun, J.-P. (2006). The contribution of qualitative analyses of occupational health and safety interventions: An example through a study of external advisory interventions. Safety Science, 44, 851-874.

Bellemare, M., Marier, M., Montreuil, S., Allard, D., \& Prévost, J. (2002). La transformation des situations de travail par une approche participative en ergonomie : une recherche intervention pour la prévention des troubles musculo-squelettiques. Études et recherches, Rapport R-292, Montréal, IRSST, 2002, 126 p. http://www.irsst.qc.ca/fr/_publicationirsst_824.html

Bourgeois, F., Lemarchand, C., Hubault, F., Brun, C., Polin, A., \& Faucheux, J-M. (2000). Troubles musculosquelettiques et travail. Quand la santé interroge l'organisation. Editions ANACT, Collection outils et méthodes.

Caroly, S. (à paraître). L'activité collective et la réélaboration de règles : des enjeux pour la santé au travail. Habilitation à Diriger des Recherches

Caroly, S., Coutarel, F., Escriva, E., Roquelaure, Y., \& Schweitzer, J.M. (2007). La prévention durable des TMS. Quels freins? Quels leviers d'action? Rapport DGT de la recherche-action 2004-2007.

Coutarel, F. (2004). La prévention des Troubles Musculo Squelettiques en conception : quelles marges de manœuvre pour le déploiement de l'activité ? Laboratoire d'Ergonomie des Systèmes complexes, Université Bordeaux 2, collection Thèses et mémoires.

Coutarel, F.,Vézina N., Berthelette D., Aublet-Cuvelier A., Descatha A., Chassaing K., Roquelaure Y., \& Ha C. (2009). Orientations pour l'évaluation des interventions visant la prévention des troubles musculosquelettiques liés au travail. Pistes, 11(2).

Daniellou, F. (1998). Une contribution au nécessaire recensement des «Repères pour s'affronter aux TMS», in F. Bourgeois (Ed.), TMS et évolutions des conditions de travail. ANACT : Études et documents, document téléchargeable à l'adresse http://www.anact.fr/sante/tms/index.html

Daniellou, F. (1999). Le statut de la pratique et des connaissances dans l'intervention ergonomique de conception. Collection Thèses et Mémoires. Editions Laboratoire d'Ergonomie des Systèmes Complexes. Université Victor Segalen Bordeaux 2. ISPED.

Denis, D., St-Vincent, M. Jetté, C., Nastasia, L., \& Imbeau, D. (2005). Les pratiques d'intervention portant sur la prévention des troubles musculo-squelettiques : un bilan critique de la littérature. Bilan de connaissances, rapport IRSST B-066, 81 p. http://www.irsst.qc.ca/fr/_publicationirsst_100130.html

Duraffourg, J., \& Vuillon, B. (Eds.) (2004). Alain Wisner et les tâches du présent-la bataille du travail réel. Toulouse: Octarès Editions.

Jeantet, A. (1998). Les objets intermédiaires dans la conception. Eléments pour une sociologie des processus de conception. Sociologie du travail, 3-98, 291-316.

Falzon, P. (1996). Des objectifs de l'ergonomie, in F. Daniellou (Ed.), L'ergonomie en quête de ses principes (pp. 232-242). Toulouse: Octarès Editions.

Franchi, P. (1997). Les maladies professionnelles : l'exemple des troubles musculosquelettiques (TMS). Lyon: Editions de l'ANACT, Collection Agir sur...

Landry, A. (2008). L'évaluation de l'intervention ergonomique : de la recherche évaluative à la proposition d'outils pour la pratique. Thèse pour le doctorat de l'université Bordeaux 2. Mention ergonomie.

Landry, A., \& Feillou, I. (2008). Positionner l'ergonomie dans la conception : exemple dans la grande distribution. P.I.S.T.E.S, 10(2). http://www.pistes .uqam.ca/v10n2/articles/v10n2a9.htm

Landry, A., \& Tran Van, A. (2010). L'évaluation, un outil de l'ergonome pour transformer le contexte d'intervention. P.I.S.T.E.S, 12(2). http://www.pistes.uqam.ca/v12n2/pdf/v12n2a3.pdf

Leplat, J. (Ed.) (1992). L'analyse du travail en psychologie ergonomique, Tome 1. Toulouse: Octarès Editions. 
Leplat, J. (2002). De l'étude de cas à l'analyse de l'activité. P.I.S.T.E.S., 4(2). http://www.pistes.uqam.ca/ $\mathrm{v} 4 \mathrm{n} 2 /$ articles/v4n $2 \mathrm{a} 8 . \mathrm{htm}$

Nahon, P., \& Arnaud, S. (2001). Interactions en chaîne. Santé et Travail, n³5, Avril.

Saint-Arnaud, Y. (1992). Connaître par l'action, Collection INTERVENIR dirigée par M. Payette, Les Presses de L'université de Montréal, Montréal.

Saint-Vincent, M., Gonella, M., Beauvais, A., Vézina, N., Laberge, M., Lévesque, J., Voulome, T., Dubé, J., Lévesque, S., \& Cole, D. (2008). L’intervention ergonomique participative pour prévenir les TMS : ce qu'en dit la littérature francophone. Actes du 43ème Congrès de la Self, Ajaccio, 17-19 septembre, pp. 449-457.

Terssac, G. de, \& Chabaud, C. (1990). Référentiel opératif commun et fiabilité, in J. Leplat, \& G. de Terssac (Eds.), Les facteurs humains de la fiabilité dans les systèmes complexes, (pp. 111-140). Toulouse: Octarès Editions.

Tran Van, A., Landry, A., \& Martin, Ch. (2008). Place des orientations stratégiques dans la conception. In Actes du 43 $3^{\text {eme }}$ congrès de la SELF, Ajaccio, pp. 419-425.

Vézina, N. (2001). La pratique de l'ergonomie face aux TMS : ouverture à l'interdisciplinarité. Compterendu du 36 ${ }^{\text {ème }}$ congrès SELF-ACE 2001, Montréal, pp.44-60.

Vinck, D. (2000). Approches sociologiques de la cognition et prise en compte des objets intermédiaires. Cours à la septième Ecole d'été de l'ARCo. Bonas, 10-21 Juillet 2000.

Volkoff, S. (coord.) (2005). L'ergonomie et les chiffres de la santé au travail ; Ressources, tensions et pièges. Toulouse: Octarès Editions.

Yin, R. (1994). Case Study Research, Design and Method, second ed. Sage Publication, Thousand Oaks.

\begin{abstract}
RÉSUMÉ
Une étude de cas multiples menée sur plusieurs interventions visant la prévention des Troubles Musculo-squelettiques (TMS) nous a permis de tester les qualités opératives de l'utilisation par les ergonomes d'un modèle de TMS pour favoriser la prévention durable dans les entreprises. Après avoir choisi un modèle de TMS présentant à la fois l'étiologie des TMS et l'intervention de prévention, selon le cadre théorique des objets intermédiaires, l'usage du modèle semblerait faciliter le dialogue entre les acteurs de l'entreprise à propos des TMS, leur fournissant une bonne connaissance du phénomène les poussant à spontanément proposer des améliorations au modèle proposé. Suite à l'usage d'un modèle de TMS, les auteurs en proposent une évolution en intégrant les nouvelles connaissances produites sur l'étiologie des TMS et en intégrant les commentaires des acteurs des entreprises.
\end{abstract}

\title{
Mots CLÉS
}

modèle opérant, objet intermédiaire, troubles musculo-squelettiques, prévention durable.

\section{RÉFÉRENCEMENT}

Buchmann, W. \& Landry, A. (2010). Intervenir sur les TMS. Un modèle des Troubles Musculo-squelettiques comme objet intermédiaire entre ergonomes et acteurs de l'entreprise. Activités 7(2), 84-103, http://www. activites.org/v7n2/v7n2.pdf.

Article soumis le 29 mars 2010, accepté pour publication le 30 août 2010. 